\title{
Multipath QoS-Driven Routing Protocol for Industrial Wireless Networks
}

\author{
Miguel Sepulcre, Javier Gozalvez, Baldomero Coll-Perales \\ msepulcre@umh.es, j.gozalvez@umh.es, bcoll@umh.es \\ UWICORE, Ubiquitous Wireless Communications Research Laboratory, www.uwicore.umh.es \\ Universidad Miguel Hernández de Elche (UMH). Avda. de la Universidad, s/n, 03202, Elche (Spain).
}

\begin{abstract}
The Industry 4.0 concept targets the interconnection and computerization of traditional industries to improve their adaptability and utilize efficiently their resources. Industrial wireless networks will play a key role within the Industry 4.0 as they will facilitate the deployment of novel industrial applications thanks to the flexible and reconfigurable wireless connection of industrial devices. Significant advances are still necessary to enable the deployment of reliable industrial wireless networks capable to guarantee the strict QoS (Quality of Service) requirements of industrial applications in harsh propagation conditions. This paper contributes towards this objective with a novel multipath routing protocol that identifies and establishes the necessary redundant routes between any pair of nodes of a wireless network in order to satisfy the reliability and delay QoS levels demanded by industrial applications. The proposed protocol is here presented and analyzed under the framework of the WirelessHART standard given its important industrial adoption. However, it can also be adapted to other centralized TDMA-based multi-hop wireless networks.
\end{abstract}

Index Terms - Industrial wireless networks, industrial wireless sensor networks, industry 4.0, factories of the future, multipath routing, QoS, reliability, delay, cyber physical systems, CPS.

\section{INTRODUCTION}

International research initiatives are currently working to define the future of industries and manufacturing under the umbrella of the Industry 4.0 concept. The Industry 4.0 concept is based on the interconnection and computerization of traditional industries (such as manufacturing) to enable smart and adaptable factories that efficiently utilize resources and integrate components and systems [1]. To this aim, the Industry 4.0 will utilize Internet of Things (IoT) and Cyber Physical Systems (CPS) within industrial production systems [2]. This vision is in line with the Factories of the Future (FoF) roadmap of the European Commission [3] that also includes networked factories as a high priority and objective.

Industrial wireless networks will play a key role in the development of the Industry 4.0 concept. Wireless technologies can reduce the cost and time needed for the installation and maintenance of cables and machinery, enhance the flexibility and reconfigurability of a factory, and enable the connectivity of mobile subsystems, devices or robots, among others [4]. Wireless technologies can enable novel applications such as the continuous monitoring of the health and status of critical equipment and processes, or the real-time tracking of goods [5]. The evolution and maturity of certain wireless technologies have resulted in an increasing interest for the introduction of wireless networks in factories. An example is the development of the WirelessHART [6] standard for industrial automation and control applications. WirelessHART is based on the IEEE 802.15.4 PHY (Physical) layer operating in the $2.4 \mathrm{GHz}$ ISM band. WirelessHART improves the reliability of wireless transmissions through the use of a centralized network management architecture, multi-channel Time Division Multiple Access (TDMA), redundant routes, and Frequency Hopping (FH) [7]. However, industrial wireless networks still face significant challenges to guarantee the strict end-to-end reliability and delay requirements of industrial applications [8].

Industrial wireless networks need to ensure that it is possible to establish routes to reliably transmit information between different nodes of the network. In WirelessHART, the network manager is responsible for the establishment and configuration of routes. To this aim, the network manager collects network topology information through regular reports sent by the network nodes, and determines the routes taking into account the reported QoS and the application requirements. WirelessHART defines two routing methods: graph routing and source routing [6][9]. Graph routing is the main routing method for data communications and source routing should be used only for testing routes, troubleshooting network paths or for routing join responses [6]. A routing graph is a directed list of paths that connect two nodes. WirelessHART creates a graph for any pair of nodes that need to communicate, and assigns a Graph_ID to each graph. The source includes the Graph_ID in each packet's network header, and intermediate nodes identify possible forwarders based on the graph identified by the Graph_ID. The network manager is responsible for creating the routing graphs (usually uplink, downlink and broadcast graphs), and for allocating the necessary time slots for each link in the route. WirelessHART creates a primary route between source and destination, and one or more alternate paths for possible retransmissions. The network manager allocates two or more dedicated slots for each link in a primary route and a shared slot on each link of the alternate path [10]. Many studies normally assume that only two slots are assigned to each link on the primary route. However, implementations with a higher number of dedicated slots for each link in the primary 
route are also possible [6]. WirelessHART introduces alternate routing paths to allow for retransmissions and increase the end-to-end reliability levels. However, recent studies (e.g. [9]) have revealed that further improvements are necessary to satisfy the strict reliability requirements of industrial applications. In this context, this paper proposes MPAR, a centralized MultiPAth QoS-driven Routing protocol for industrial wireless networks. MPAR has been designed to identify the redundant routes that need to be established between any source and destination nodes in order to satisfy the end-to-end reliability and delay requirements demanded by industrial applications. To this aim, MPAR uses the information collected by the network manager to estimate the end-to-end reliability and delay performance of multi-hop routes. MPAR is here presented and analyzed under the framework of the WirelessHART standard given its important industrial adoption. However, MPAR can also be adapted to other centralized TDMA-based multi-hop wireless networks. Similarly, the design of MPAR could also be driven by other metrics than reliability and delay.

The rest of this paper is organized as follows. Section II describes the state of the art related to single-path and multipath routing protocols. Section III presents the metrics that are utilized by MPAR to estimate the end-to-end reliability and delay of multi-hop routes, and Section IV describes the MPAR protocol. MPAR is evaluated in Section $\mathrm{V}$, and Section VI summarizes the main contributions and conclusions of this study.

\section{STATE OF THE ART}

Multiple routing protocols have been proposed in the literature to find the optimal multi-hop route between two nodes in a network that satisfies certain end-to-end QoS requirements (e.g. throughput, reliability or delay). The QOLSR (QoS Optimized Link State Routing) protocol [11] assigns weights to individual links based on average delay and bandwidth metrics, and then finds the optimal path in terms of bandwidth and delay between source and destination. InRout [12] is a distributed multi-metric routing protocol that looks for the optimum route between two nodes. InRout uses Q-learning to choose the optimum routes taking into account current network conditions (packet error rate - PER, energy and buffer capacity) and application settings. The optimum route is the one that includes the nodes with highest energy levels, and that achieves an estimated PER below the application's PER requirement. QMRP [13] is a QoS multicast routing protocol for partially mobile wireless TDMA networks. The objective of QMRP is to provide predictable communication bandwidths and transfer delays in industrial wireless networks. To this aim, QMRP iteratively constructs routing trees and reserves time slots along the branches of these trees. QMRP is able to guarantee bandwidth and delay QoS requirements by assigning dedicated slots. Other routing protocols focus on minimizing the delay between source and destination. For example, the AAQR (Application Aware QoS Routing) protocol [14] measures the transmission time of each individual wireless link using RTP (Real-time Transport Protocol), and compares it with the application's delay requirements to select the best route between source and destination. The proposal in [15] exploits the geographic position of nodes to select and prioritize the routes depending on energy efficiency, delay, and complexity.

Selecting the optimal route in a network based on certain metrics does not necessarily result in that the selected route is capable to satisfy the end-to-end QoS requirements. To improve the end-to-end QoS, several approaches have been reported. For example, [16] proposes to reduce end-to-end delay by allowing intermediate nodes to forward the received packets in the same time slot they are received if there is sufficient time to do so. This approach avoids the delay resulting from traditional schemes where the packet to be forwarded would be buffered until the next transmitting slot. A different alternative to improve the end-to-end QoS is the establishment and use of multiple paths or routes between source and destination. In [17], the proposed multipath routing protocol uses backup routes as alternate routes when the main one fails. Backup routes are created at the same time than the primary route, but are not used unless at least one link of the primary route fails. More recent multipath routing proposals simultaneously exploit multiple paths by splitting the information to be transmitted between different routes. In this case, the reliability between source and destination increases with the number of utilized paths. For example, [18] proposes a delay-differentiated multi-speed packet forwarding strategy together with an in-node packet scheduling mechanism to improve the reliability, delay and energy efficiency of end-to-end connections. The work in [19] presents an on-demand Multipath Source Routing protocol (MSR). During the routing discovery phase, RREQ (Route Request) and RREP (Route Reply) messages are used to identify the routes that satisfy the bandwidth requirements (i.e. the routes in which all links have sufficient available bandwidth). Then, a pre-defined number of routes are used to transmit the information in order to achieve higher reliability. Other protocols such as [20] propose the use of adaptive traffic distribution schemes to distribute the traffic in a multipath route so that the end-to-end delay is minimized. The identification and selection of the best multipath routes is normally based on the estimation of certain metrics. Studies such as [21] and [22] develop analytical models to estimate the end-to-end reliability, delay or throughput based on 1-hop metrics. In particular, [21] and [22] develop an analytical model to estimate the probability that the number of packets lost in a multipath transmission does not exceed a threshold $M$. The authors then reconstruct the original $N$ packets at the destination using diversity coding.

The multipath routing protocols reported in the literature are generally aimed at maximizing or minimizing certain endto-end performance metrics, but do not provide any guarantees that the obtained solution is able to satisfy the objective QoS metrics, in our case the reliability and delay requirements demanded by industrial applications [23]. This is especially critical for industrial wireless networks that need to support the strict QoS requirements of industrial applications under harsh propagation conditions in factories. In this context, this paper proposes MPAR, a multipath routing protocol that establishes end-to-end connections between source and destination nodes using the necessary 
redundant routes to satisfy the reliability and delay requirements imposed by the applications. MPAR is designed for wireless networks where a central entity manages the establishment of routes between any nodes in the network; this is for example the case of industrial wireless networks like WirelessHART that include a network manager. MPAR uses link performance metrics reported by each network node to the central entity. The central entity uses these metrics to estimate the end-to-end reliability and delay, and identify and establish the necessary redundant routes between source and destination nodes to guarantee the reliability and delay application requirements.

\section{QOS METRICS FOR MULTIPATH ROUTE SELECTION}

The MPAR proposal requires estimating the end-to-end reliability and delay performance to determine the number of multipath source-destination routes necessary to satisfy the QoS requirements demanded by the application, select such routes and identify the forwarding nodes. This section analytically derives the end-to-end reliability and delay QoS performance metrics that are utilized by the network manager to evaluate candidate multipath routes. Both metrics depend on factors such as the number of hops and the quality of each wireless link, and therefore are not independent. The objective of the derived metrics is to provide higher QoS guarantees than simply estimating average reliability and delay values.

\section{A. Reliability}

The reliability of an end-to-end connection can be defined as the probability that a packet generated at the source node successfully arrives to the destination. Factors influencing the end-to-end reliability include the reliability of each 1-hop wireless link, the maximum number of retransmissions that are possible at each hop, and the number of multiple redundant routes between source and destination.

The transmission of a packet over a 1-hop wireless link between certain nodes $a$ and $b$ can be represented by a Bernoulli trial with probability of success $p_{a b}$, where $p_{a b}$ is equivalent to the probability of correct packet reception or PDR (Packet Delivery Ratio). Given the probabilistic nature of radio propagation, multiple retransmissions might be needed to successfully deliver a packet in the link. As a result, it is necessary to take into account that each packet can be retransmitted up to $n_{\max }$ times in each link (one transmission plus $n_{\max }-1$ retransmissions). If we assume that transmission errors are independent (i.e. propagation errors do not occur in bursts), the process can be represented by a Bernoulli experiment consisting in $n_{\max }$ statistically independent Bernoulli trials. The number of successes has a binomial distribution $B\left(n_{\max }, p_{i j}\right)$. The reliability of a 1-hop link can be estimated as the inverse of the probability that $n_{\max }$ consecutive transmissions fail, i.e. as the inverse of the probability that $k=0$ successes occur in the $n_{\max }$ experiments:

$$
P_{a b}=1-\left(\begin{array}{c}
n_{\max } \\
k
\end{array}\right) p_{a b}^{k}\left(1-p_{a b}\right)^{n_{\max }-k}=1-\left(1-p_{a b}\right)^{n_{\max }}
$$

Packet retransmissions can be considered independent of each other in scenarios in which the channel coherence time is lower than the time between retransmissions. This is a reasonable assumption for this study since WirelessHART utilizes TDMA as multiple access scheme. If we consider that a single-path route, $r$, between source and destination is composed of $L$ independent wireless links, it is possible to estimate its end-to-end reliability as follows:

$$
P^{r}=\prod_{i=1}^{L} P_{i[i+1]}=\prod_{i=1}^{L}\left(1-\left(1-p_{i[i+1]}\right)^{n_{\max }}\right)
$$

where $p_{i[i+1]}$ represents the PDR of a wireless link between two consecutive nodes in the route.

Multiple independent redundant routes between a pair of source and destination nodes can improve the reliability of their end-to-end connection. In this case, a packet will be successfully delivered to the destination when it is correctly transmitted through at least one of the established routes (even if the rest of the routes fail in delivering the same packet to the destination node). If we consider $R$ redundant and independent routes between source and destination nodes, the end-to-end reliability can be estimated as follows:

$$
P=1-\prod_{r=1}^{R}\left(1-P^{r}\right)=1-\prod_{r=1}^{R}\left(1-\prod_{i=1}^{L}\left(1-\left(1-p_{i[i+1]}\right)^{n_{\max }}\right)\right)
$$

where $\left(1-P^{r}\right)$ represents the probability that a packet is not correctly received at the destination through route $r$.

\section{B. Delay}

The end-to-end delay between a pair of source and destination nodes that are connected via multiple redundant routes can be defined as the minimum of the time it takes a packet to reach the destination using each one of the multiple routes. Estimating the end-to-end delay requires first quantifying the delay that a packet experiences in each of the 1-hop links that are part of a source-destination route. Such delay depends on the number of retransmissions needed to successfully transmit the packet. We define $n_{a b}$ as the random variable that represents the number of packet retransmissions needed to successfully transmit one packet from node $a$ to node $b$. We also define $\tau_{T}$ as the packet transmission duration, and $\tau_{R}$ as the time between consecutive packet retransmissions. The delay experienced by a packet in the wireless link between nodes $a$ and $b$ can then be modeled as ${ }^{1}$ :

$$
\delta_{a b}=\tau_{T}+\tau_{R} n_{a b}
$$

The number of retransmissions, $n_{a b}$, depends on the probability that a packet transmitted by node $a$ is correctly received by node $b$. In general, this probability depends on the propagation conditions between nodes $a$ and $b$ for a TDMAbased system. Considering that a packet transmission can be represented by a Bernoulli trial with probability of success $p_{a b}$, the number of retransmissions needed, $n_{a b}$, is equivalent to the number of Bernoulli trials needed to obtain one success. $n_{a b}$ follows then a geometric distribution, and the probability

\footnotetext{
${ }^{1}$ Please note that this study does not consider the time between the packet generation and the transmission slot.
} 
that the number of packet retransmissions in a link is exactly $n$ can be calculated as:

$$
\operatorname{Pr}\left\{n_{a b}=n\right\}=p_{a b}\left(1-p_{a b}\right)^{n}
$$

As it was previously considered when deriving eq. (2), this result also considers that packet retransmissions are independent of each other. Following eq. (5), the probability that a packet is correctly received without any retransmission is equal to $\operatorname{Pr}\left\{n_{a b}=0\right\}=p_{a b}$, and the delay $\delta_{a b}$ is equal to $\tau_{T}$. The expected value of the number of retransmissions is $E\left(n_{a b}\right)=(1$ $\left.p_{a b}\right) / p_{a b}$. The distribution function of the number of needed packet retransmissions follows the distribution function of a geometric random variable and can be expressed as:

$$
F\left\{n_{a b}=n\right\}=\operatorname{Pr}\left\{n_{a b} \leq n\right\}=1-\left(1-p_{a b}\right)^{n+1}
$$

The distribution function describes the probability that the number of packet retransmissions $n$ is lower than or equal to a certain value. Therefore, the distribution function in equation (6) can be used to calculate the maximum number of retransmissions $n_{\alpha}$ that will be needed in a link with certain probability $\alpha$. That is, the number of packet retransmissions will be lower than or equal to $n_{\alpha}$ with probability $\alpha$, and $n_{\alpha}$ can be calculated as:

$$
\alpha=1-\left(1-p_{a b}\right)^{n_{\alpha}+1} \Rightarrow n_{\alpha}=\frac{\log (1-\alpha)}{\log \left(1-p_{a b}\right)}-1
$$

Eq. (7) can then be used to estimate the maximum delay that a packet could experience in a 1-hop wireless link with probability $\alpha$ :

$$
\delta_{a b}=\tau_{T}+\tau_{R}\left(\frac{\log (1-\alpha)}{\log \left(1-p_{a b}\right)}-1\right)
$$

The end-to-end delay in a single-path route consisting of $H$ wireless hops can then be expressed as:

$$
\delta_{1[1+H]}=\tau_{T} H+\tau_{R} n_{1[1+H]}=\tau_{T} H+\tau_{R} \sum_{i=1}^{H} n_{i[i+1]}
$$

where $n_{i[i+1]}$ represents the number of packet retransmissions in the $i^{\text {th }}$ hop in the route, i.e. between nodes $i$ and $i+1$. To estimate the end-to-end delay, it is first necessary to estimate the total number of retransmissions needed in the complete single-path route to successfully transmit a packet between source and destination nodes. This number can be modeled as a random variable equal to the sum of the number of packet retransmissions of all the 1-hop links forming the route. The distribution function of a random variable that is the sum of independent random variables can be obtained as the convolution of the distribution functions of the independent random variables. As a result, the probability distribution of the number of packets retransmissions needed for a singlepath route with two hops $\left(n_{a c}=n_{a b}+n_{b c}\right)$ can be expressed as:

$$
\operatorname{Pr}\left\{n_{a c}=n\right\}=\sum_{i=0}^{n} \operatorname{Pr}\left\{n_{a b}=i\right\} \operatorname{Pr}\left\{n_{b c}=n-i\right\}
$$

Eq. (10) can be further developed as follows:

$$
\begin{aligned}
& \operatorname{Pr}\left\{n_{a c}=n\right\}=\sum_{i=0}^{n} p_{a b}\left(1-p_{a b}\right)^{i} p_{b c}\left(1-p_{b c}\right)^{n-i}= \\
& =p_{a b} p_{b c}\left(1-p_{b c}\right)^{n} \sum_{i=0}^{n}\left(\frac{1-p_{a b}}{1-p_{b c}}\right)^{i}= \\
& =\frac{p_{a b} p_{b c}}{p_{a b}-p_{b c}}\left(\left(1-p_{b c}\right)^{n+1}-\left(1-p_{a b}\right)^{n+1}\right)
\end{aligned}
$$

Similarly, the probability distribution of the number of packets retransmissions needed for a route with three hops $\left(n_{a d}=n_{a b}+n_{b c}+n_{c d}\right)$ can be modeled as:

$$
\begin{aligned}
& \operatorname{Pr}\left\{n_{a d}=n\right\}=\sum_{i=0}^{n} \operatorname{Pr}\left\{n_{a c}=i\right\} \operatorname{Pr}\left\{n_{c d}=n-i\right\}= \\
& =\sum_{i=0}^{n} \frac{p_{a b} p_{b c}}{p_{a b}-p_{b c}}\left(\left(1-p_{b c}\right)^{i+1}-\left(1-p_{a b}\right)^{i+1}\right) p_{c d}\left(1-p_{c d}\right)^{n-i}= \\
& =\frac{p_{a b} p_{b c} p_{c d}\left(p_{B C}-p_{A B}\right)\left(1-p_{C D}\right)^{n+2}}{\left(p_{a b}-p_{b c}\right)\left(p_{b c}-p_{c d}\right)\left(p_{c d}-p_{a b}\right)}+ \\
& +\frac{p_{a b} p_{b c} p_{c d}\left(p_{A B}-p_{C D}\right)\left(1-p_{B C}\right)^{n+2}}{\left(p_{a b}-p_{b c}\right)\left(p_{b c}-p_{c d}\right)\left(p_{c d}-p_{a b}\right)}+ \\
& +\frac{p_{a b} p_{b c} p_{c d}\left(p_{C D}-p_{B C}\right)\left(1-p_{A B}\right)^{n+2}}{\left(p_{a b}-p_{b c}\right)\left(p_{b c}-p_{c d}\right)\left(p_{c d}-p_{a b}\right)}
\end{aligned}
$$

This result can be extended to obtain the probability distribution of the number of packets retransmissions needed for a single-path route with $H$ hops:

$$
\operatorname{Pr}\left\{n_{1[1+H]}=n\right\}=(-1)^{n-1} \prod_{i=1}^{H} p_{i[i+1]} \sum_{j=1}^{H} \frac{\left(1-p_{j[j+1]}\right)^{n+H-1}}{\prod_{\substack{k=1 \\ k \neq j}}^{H}\left(p_{j[j+1]}-p_{k[k+1]}\right)}
$$

where $p_{j[j+1]}$ represents the probability of packet reception between node $j$ and the next node in the route. From equation (13), the distribution function of the number of packets retransmissions needed for a single-path route can be expressed as:

$$
\begin{aligned}
& F\left\{n_{1[1+H]}=n\right\}=\operatorname{Pr}\left\{n_{1[1+H]} \leq n\right\}=\sum_{m=0}^{n} \operatorname{Pr}\left\{n_{1[1+H]}=m\right\}= \\
& =(-1)^{n-1} \prod_{i=1}^{H} p_{i[i+1]} \sum_{j=1}^{H} \frac{\left(1-p_{j[j+1]}\right)^{H-1}\left(1-\left(1-p_{j[j+1]}\right)^{n+1}\right)}{\prod_{\substack{k=1 \\
k \neq j}}^{H} p_{j[j+1]}\left(p_{j[j+1]}-p_{k[k+1]}\right)}
\end{aligned}
$$

Given the linear relationship between delay and number of packet retransmissions in eq. (9), eq. (14) can be used to model the distribution function of the end-to-end delay in a single-path route:

$$
F\left\{\delta_{1[1+H]}=\tau_{T} H+\tau_{R} n\right\}=F\left\{n_{1[1+H]}=n\right\}
$$


This study considers that MPAR simultaneously transmits every packet through the various routes established between source and destination. This is done to increase the end-to-end reliability and be able to guarantee the strict QoS requirements of industrial applications. In this case, the endto-end delay experienced by a packet transmitted over the $R$ routes that make up the multipath route is equal to the minimum delay of the different routes:

$$
\delta=\min \left(\delta_{1\left[1+H_{1}\right]}^{1}, \delta_{1\left[1+H_{2}\right]}^{2}, \delta_{1\left[1+H_{3}\right]}^{3}, \ldots, \delta_{1\left[1+H_{R}\right]}^{R}\right)
$$

where $\delta_{1\left[1+H_{j}\right]}^{j}$ represents the delay in route $j^{\text {th }}$. The end-to-end delay is then a random variable equal to the minimum of $R$ random variables. It can be demonstrated that the distribution function can then be expressed as:

$$
F\{\delta\}=1-\prod_{r=1}^{R}\left(1-F\left\{\delta_{1\left[1+H_{r}\right]}^{r}=\delta\right\}\right)
$$

The distribution function of the end-to-end delay presented in eq. (17) can be used to estimate the maximum delay that a packet would experience in a multipath route with certain probability. For example, eq. (17) can be used to estimate the maximum end-to-end delay that could be experienced in a given multipath route with a probability of e.g. $\beta=0.95$. If such delay is lower than the delay demanded by the application, the multipath route can satisfy the delay requirements of the application with a probability of 0.95 .

\section{Validation}

Sections III.A and III.B have presented analytical models to estimate the end-to-end reliability and delay of multipath routes. This section reports the validation of these models through extensive simulations. The validation is here illustrated considering a scenario where the source and destination nodes are connected through 4 independent routes. Each packet is transmitted by the source node through the four identified routes. The packet is correctly received at the destination if it reaches the destination node through at least one of the routes. The delay of a packet correctly received at the destination is the minimum delay experienced through the different routes. The four routes have 5, 5, 4 and 3 hops, respectively. Each hop in the route has been randomly assigned a PDR value reported in Table I.

Fig. 1 compares the end-to-end reliability estimated using our proposed analytical model with the average reliability computed through the simulations. The figure differentiates between the reliability obtained using each route individually, and the reliability obtained when transmitting packets simultaneously through all four routes. The results show a close match between the reliability estimates using the analytical model and the simulations, especially when the number of packets transmitted increases. Fig. 1 also illustrates the reliability benefits that can be obtained using multipath routes compared to single-path ones. The results reported in Fig. 1 have been obtained considering that the maximum number of transmissions and retransmissions per hop is $n_{\max }=4$ ( 1 transmission and 3 retransmissions).

Fig. 2 compares the distribution function of the end-to-end delay estimated using our proposed analytical model and computed through the simulations. In this figure, the $x$-axis represents the end-to-end delay $(\delta)$. For simplification, the packet transmission duration $\left(\tau_{T}\right)$ and the time between consecutive packet retransmissions $\left(\tau_{R}\right)$ have been set to 1 . As a result, a unit in the $x$-axis of Fig. 2 represents the delay associated with either a packet transmission or a retransmission. For example, $\delta=5$ means that a packet was transmitted from source to destination in the time equivalent to 5 transmissions and retransmissions $\left(\tau_{T}\right.$ and $\tau_{R}$ are both set equal to one). The $y$-axes represents the cumulative distribution function of the end-to-end delay $(F(\delta))$. For example, $F(5)=0.3$ means that $30 \%$ of the packets required the time equivalent to 5 or less transmissions and retransmissions to reach their destination. Fig. 2 shows that the analytical model closely matches the simulation results. The figure also shows that the end-to-end delay can be considerably reduced when utilizing multipath routes compared to single-path ones. The simulated distribution functions have been obtained estimating the delay experienced by 2000 packets transmitted from the source node to the destination one.

TABLE I

PDR VALUES FOR EACH HOP AND ROUTE IN THE VALIDATION SCENARIO

\begin{tabular}{llllll}
\hline \hline Route & Hop 1 & Hop 2 & Hop 3 & Hop 4 & Hop 5 \\
\hline 1 & 0.54 & 0.59 & 0.31 & 0.90 & 0.50 \\
2 & 0.81 & 0.92 & 0.84 & 0.77 & 0.43 \\
3 & 0.48 & 0.39 & 0.76 & 0.56 & \\
4 & 0.78 & 0.40 & 0.20 & & \\
\hline \hline
\end{tabular}

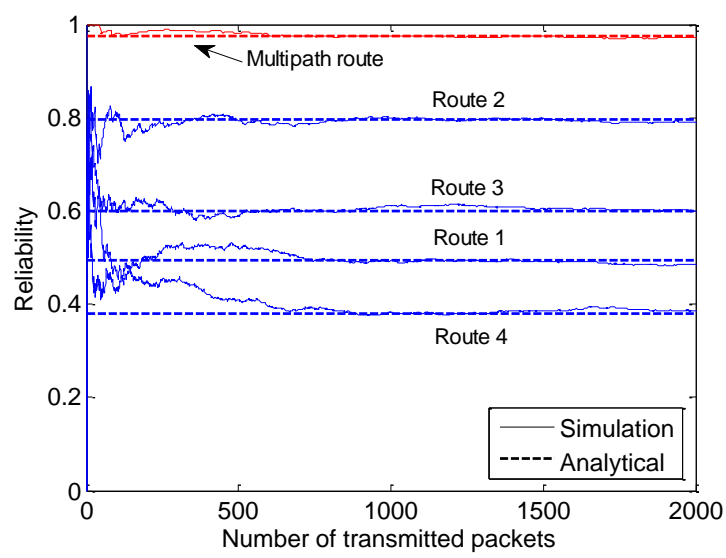

Fig. 1. Estimated end-to-end reliability using the analytical model or simulations. The results are obtained for the scenario in Table I.

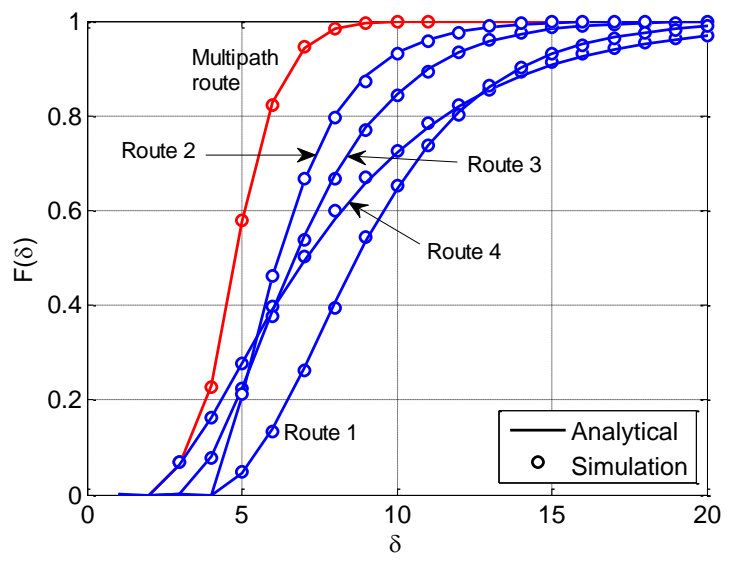

Fig. 2. Distribution function of the end-to-end delay obtained analytically and through simulations. The results are obtained for the scenario in Table I. 


\section{MultiPATH QOS-DRIVEN RouTING PROTOCOL}

The MPAR proposal has been designed to first detect whether it is possible or not to establish a multipath route capable to guarantee the end-to-end reliability and delay requirements demanded by applications. If this is possible, MPAR identifies the multipath route needed to satisfy such requirements. The proposal is built within the framework of the industrial WirelessHART standard, and hence considers that the network is managed by a centralized entity (the network manager) that is in charge of executing MPAR to establish multipath routes between any two nodes of the network. In WirelessHART, the network manager periodically collects health reports sent by the network nodes. Health reports include for each pair of neighbors statistics about the packets transmitted and received, the average received signal level, and the timestamp of the last communication with the neighbor [7]. This study proposes to use these reports to derive the probability of correct packet reception or PDR experienced by a node with each one of its neighboring nodes. This information is used by the network manager to compute the end-to-end reliability and delay metrics using the models presented in Section III, and identify the redundant routes (number of redundant routes and identity of nodes belonging to each route) needed to satisfy the QoS requirements demanded by the application. The necessary redundant routes are identified one by one in an iterative process until a multipath route capable to satisfy the QoS requirements can be identified. Fig. 3 depicts the flow chart for the MPAR proposal that operates as follows:

1. Route identification. When the network manager receives a request to establish a connection between two nodes, it first identifies the best single-path route between the source and destination nodes using the Dijkstra algorithm. The input to the Dijkstra algorithm is the graph associated to the network, where each network node is a vertex, and each edge connecting two nodes is characterized by the delay estimated using eq. (8). The output of the Dijkstra algorithm is the route between source and destination that minimizes the sum of the individual delays of each link. This route is considered to be part of the multipath route that will communicate the source and destination nodes.

2. Estimate reliability and delay. The network manager then estimates the end-to-end reliability and delay that the source and destination nodes would experience if utilizing the identified multipath route; the multipath route will be made up of a single route in the first iteration. The end-to-end reliability is calculated using eq. (3) and the end-to-end delay is computed with eq. (17) for a pre-established probability $\beta$.

3. End or new iteration. The end-to-end reliability and delay computed in step 2 for the identified multipath route are compared to the QoS requirements demanded by the application. If the identified multipath route is able to guarantee these requirements, the iterative process finishes and the network manager establishes the connection between source and destination using the identified multipath route. If the identified multipath route is not able to satisfy the application requirements, and the number of routes that make up the multipath route is below $R_{\max }$, a new iteration of the algorithm is executed and a new route is added to the multipath one. To this aim, three different approaches are possible:

3.a. Non-disjoint (NonD) routes can have nodes and links in common. With this approach, the following iteration simply selects the next best route using the Dijkstra algorithm using the same graph as in the previous iteration. To this aim, MPAR uses the generalization of the Dijkstra algorithm to find the $K$ shortest paths.

3.b. Link disjoint (LinkD) routes have no links in common, but may have nodes in common. With this approach, the links that are already part of the identified multipath route are removed from the graph before running the Dijkstra algorithm in the next iteration.

3.c. Node disjoint (NodeD) routes, or totally disjoint routes, have no nodes or links in common. With this approach, the nodes that are already part of the identified multipath route are removed from the graph before running the Dijkstra algorithm in the next iteration.

In the current version of the WirelessHART standard, the graphs built by the network manager can have multiple paths. However, simultaneous transmissions through multiple paths are not possible in WirelessHART because packets traveling along a graph can only be forwarded at each point in time to one of the neighbors belonging to the graph. MPAR requires the ability to simultaneously transmit packets over different paths between source and destination in order to minimize the end-to-end delay. MPAR would hence be compatible with WirelessHART if minor modifications are made. In particular it should be allowed that packets traveling along a graph are simultaneously forwarded to several neighbors in the graph following the routing decisions of the protocol.

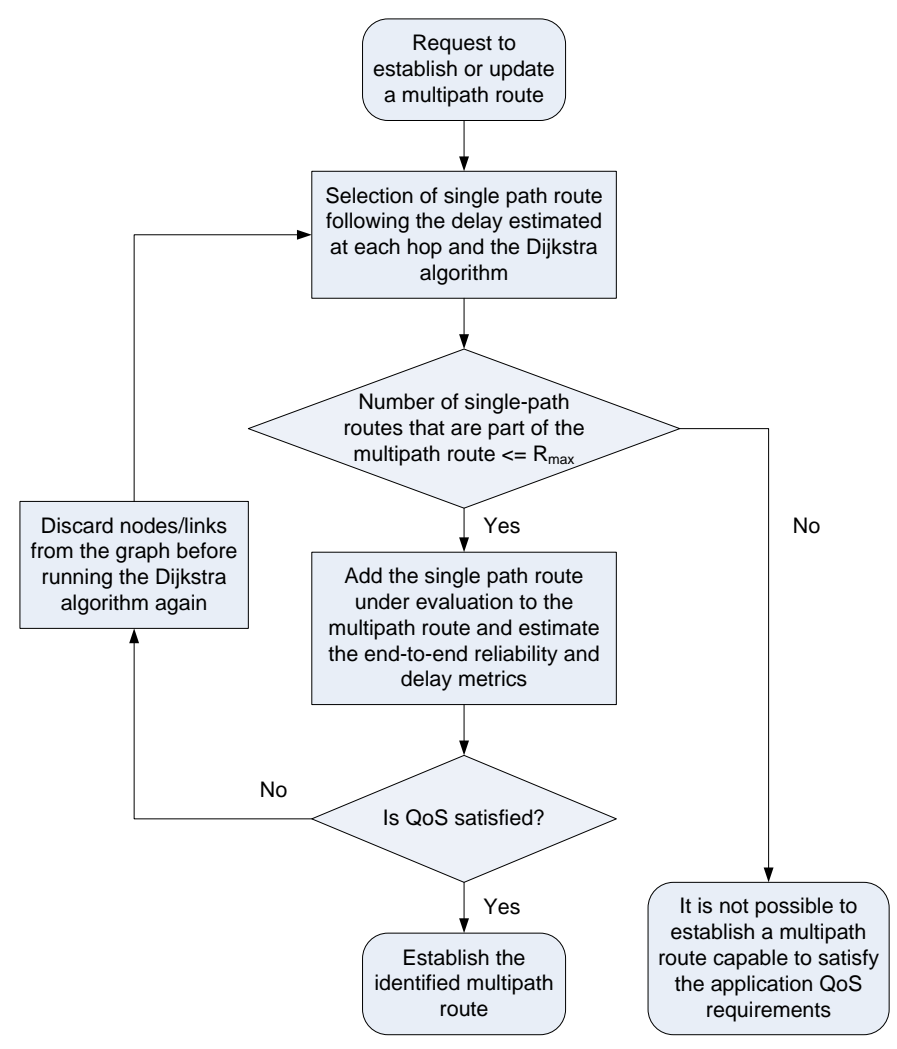

Fig. 3. MPAR flow chart. 


\section{EVALUATION}

\section{A. Scenario and settings}

The MPAR proposal has been evaluated by means of Matlab simulations where nodes are randomly and uniformly distributed over an area of 200mx200m. This study considers the WirelessHART industrial framework, and therefore assumes that the network manager periodically collects information reported by all nodes through health reports. Using this information, the network manager estimates the PDR values of all possible links. The network manager is located at the center of the scenario. During the simulations, nodes will sequentially request to establish a connection with the network manager in order to support a monitoring and control application where real-time communication is critical. Shocks, vibrations, tilts, as well as environmental parameters (temperature, pressure...) are strategic information that needs to be collected in real-time at the heart of production tools in complex and harsh environments [24]. Industrial wireless networks could be also used to periodically report relevant control parameters such as rotation speed and acceleration of production tools, and could enable the adaptive control and plug and produce capability of machine-tools during its interaction with the cutting process [25].

The network manager runs the MPAR protocol to establish the necessary routes in order to satisfy the QoS requirements imposed by an application. This study considers a generic application in order to be able to test the performance of the algorithms under different scenarios and QoS requirements. Such requirements are generally set to very demanding levels given the critical nature of most industrial processes. Once a connection is established, the source node transmits a large number of packets of 133 bytes $^{2}$ towards the network manager. The number of transmitted packets has been selected to ensure the statistical accuracy of the simulation results, and in particular a relative error below $1 \%$. The network manager measures the reliability and delay experienced using the transmitted packets, and evaluates whether the identified routes can satisfy the application requirements. The process is repeated for all nodes in the scenario.

This study uses the large-scale fading model reported in [26] for the $2.4 \mathrm{GHz}$ frequency band. The model represents challenging industrial radio propagation conditions in a scenario with obstructed line-of-sight between the transmitter and the receiver due to the presence of industrial inventory. The employed model considers log-distance path-loss and log-normal shadowing effects with the following parameters: $d_{0}=15 \mathrm{~m}$ (reference distance), $P L\left(d_{0}\right)=63.57 \mathrm{dBm}$ (path-loss at the reference distance), $n=4.29$ (path-loss exponent) and $\sigma=8.42 \mathrm{~dB}$ (shadowing standard deviation). Fig. 4 depicts the PDR (Packet Delivery Ratio) obtained using the model published in [26] with a transmission power of $3 \mathrm{~dB}$ and a reception threshold of $-80 \mathrm{dBm}$.

Table II presents the main simulation and communication parameters used in this study, including e.g. the maximum number of transmissions and retransmissions per hop $\left(n_{\max }=4\right)$

\footnotetext{
2 This is the maximum number of bytes that can be transmitted in a WirelessHART link.
}

and the maximum number of routes in a multipath route $\left(R_{\max }=7\right)$. To simplify the computation of end-end-delays, $\tau_{T}$ and $\tau_{R}$ have been set to 1 , and the application delay requirements are defined in integer units.

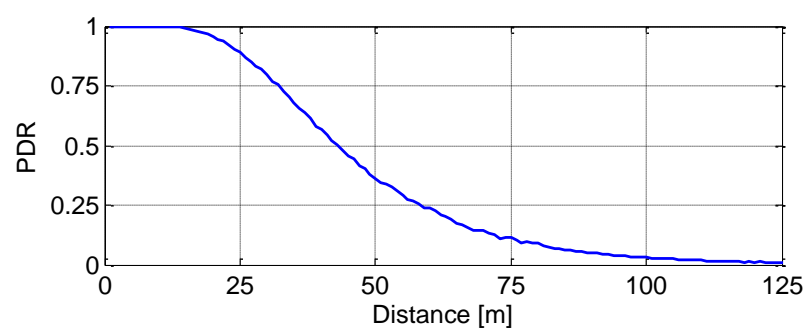

Fig. 4. PDR as a function of the distance between transmitter and receiver.

TABLE II

\begin{tabular}{llll}
\multicolumn{4}{c}{ SIMULATION AND COMMUNICATION PARAMETERS } \\
\hline & Parameter & Description & Value \\
\hline Propagation & $d_{0}$ & Reference distance & $15 \mathrm{~m}$ \\
model & $P L\left(d_{0}\right)$ & Propagation loss at $d_{0}$ & $63.57 \mathrm{~dB}$ \\
& $n$ & Pathloss exponent & 4.29 \\
& $\sigma$ & Shadowing standard & 8.42 \\
& & deviation & \\
\hline Transceiver & $P_{t}$ & Tx power & $3 \mathrm{dBm}$ \\
& $R_{t h}$ & Rx threshold & $-80 \mathrm{dBm}$ \\
& $G$ & Antenna gain & $0 \mathrm{dBi}$ \\
\hline Protocol & $\alpha$ & Delay prob. (1 hop) & 0.95 \\
parameters & $\beta$ & Delay prob. (end-to-end) & 0.95 \\
& $n_{\max }$ & Max \# of retransmissions & 4 \\
& $R_{\max }$ & Max \# of routes & 7 \\
& $\tau_{T}$ & Transmission time & 1 \\
& $\tau_{R}$ & Time between retx. & 1 \\
\hline Application & $P_{\text {req }}$ & Required reliability & 0.99 to 0.9999 \\
requirements & $\delta_{\text {req }}$ & Required delay & $6,7,8$ \\
\hline Simulation & $N_{r}$ & \# of runs & 100 \\
& $N_{n}$ & \# of nodes & $50,100,150$ \\
& $N_{p}$ & \# of packets tx per node & $10^{4}$ \\
\hline
\end{tabular}

\section{B. MPAR performance}

Fig. 5 and Fig. 6 show the end-to-end reliability and delay obtained with MPAR for different application requirements and number of deployed nodes. The MPAR proposal has been evaluated considering very high reliability requirements due to the critical nature of industrial applications. The results in Fig. 5 and Fig. 6 are obtained considering the NonD approach in MPAR. The bars in the figures represent average values, and the vertical ones the $5 \%$ and $95 \%$ percentiles. For a fixed reliability requirement $\left(P_{\text {req }}=0.99\right)$, Fig. 5 shows that the connections established by MPAR could satisfy the reliability requirements for $\delta_{r e q}=6,7$ and 8. Similarly, Fig. 6 shows that the delay requirements are also satisfied by MPAR for $P_{\text {req }}=0.99$ to 0.9999 and $\delta_{r e q}=8$. These results demonstrate that MPAR can identify and establish the multipath routes needed to satisfy the reliability and delay requirements demanded by applications. Very similar end-to-end reliability and delay results have been obtained with the LinkD and NodeD approaches. 


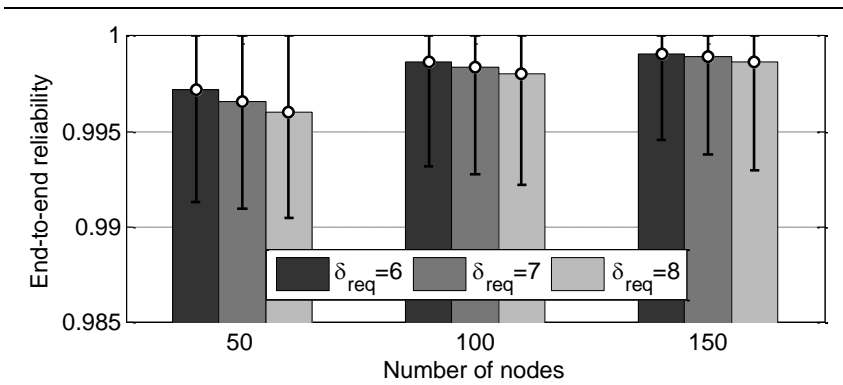

Fig. 5. End-to-end reliability obtained with MPAR (NonD approach) for $\mathrm{P}_{\text {req }}=0.99$.

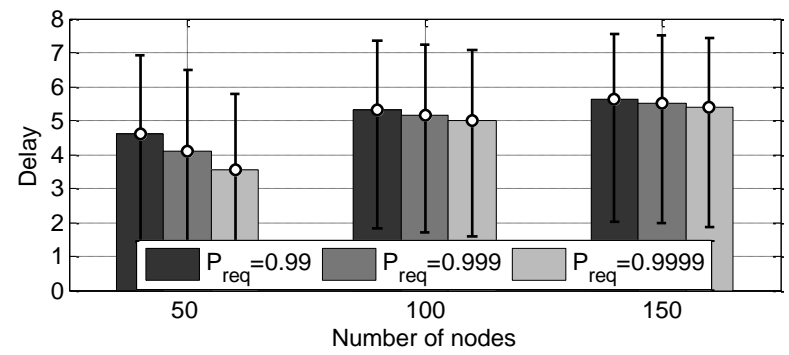

Fig. 6. End-to-end delay obtained with MPAR (NonD approach) for $\delta_{\text {req }}=8$.

One of the key benefits of MPAR is its capability to adapt to the application requirements. MPAR is able to establish the necessary redundant routes between source and destination nodes to satisfy the requirements demanded by the application. Fig. 7 shows again the end-to-end reliability obtained with MPAR but for $P_{r e q}=0.999$. The comparison of Fig. 5 and Fig. 7 shows that MPAR is able to adapt its operation to the higher reliability requirements in Fig. 7, and create the necessary routes to satisfy such requirements independently of the number of nodes and the required delay. Similarly, Fig. 8 shows the end-to-end delay obtained with MPAR for $\delta_{\text {req }}=7$. The comparison of Fig. 6 and Fig. 8 shows again that MPAR is also able to adapt its operation to a change in the required delay, and still satisfy the application requirements. MPAR is able to satisfy changing application requirements by adapting the number of redundant routes used per end-to-end connection. Fig. 9 illustrates the influence of the application requirements on the number of necessary redundant routes. The bars represent average values, and the vertical lines the 5\% and 95\% percentiles. Fig. 9 shows that the number of routes needed to satisfy the application requirements increases with such requirements. For example, MPAR established 2.54 redundant routes in average between source and destination when the reliability requirement was equal to 0.99 and the delay was equal to 7 (Fig. 5). When the reliability requirement increases to 0.999 (Fig. 7 and a delay requirement of 7), MPAR adapts its operation and establishes 3.12 redundant routes in average between source and destination to meet the more strict QoS requirements. Fig. 9 also shows that the number of routes needed to satisfy the application requirements decreases with the number of nodes in the scenario. This is the case because the probability to establish reliable wireless links with neighbor nodes increases with the nodes' density. MPAR can meet the application requirements with less redundant routes between source and destination when more reliable wireless links can be established between neighboring nodes.

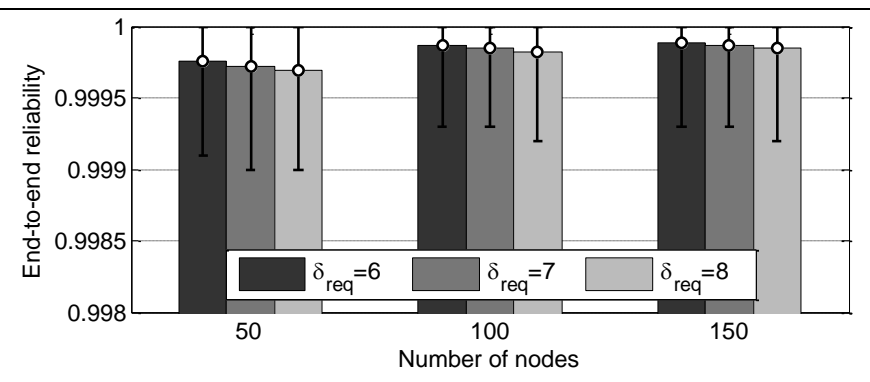

Fig. 7. End-to-end reliability obtained with MPAR (NonD approach) for $\mathrm{P}_{\mathrm{req}}=0.999$.

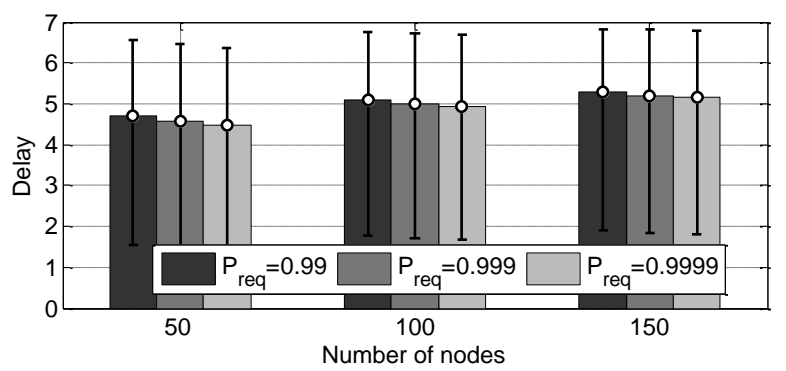

Fig. 8. End-to-end delay obtained with MPAR (NonD approach) for $\delta_{\text {req }}=7$.

Fig. 10 shows that the number of routes needed by MPAR highly depends on the position and distance between source and destination nodes. The results depicted in Fig. 10 show that the highest number of routes is required for the source nodes located at the corners of the scenario; the destination node (the network manager) is located at the center of the scenario. Fig. 10 also demonstrates that MPAR is capable to adapt its operation (number of redundant routes) to the connectivity conditions between source and destination nodes. These conditions are highly related to the distance between source and destination.

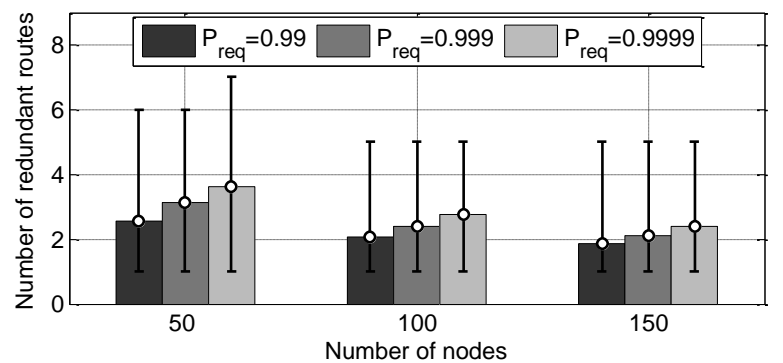

Fig. 9. Number of redundant routes needed by MPAR (NonD approach) to guarantee the reliability requirements for $\delta_{\mathrm{req}}=7$. 


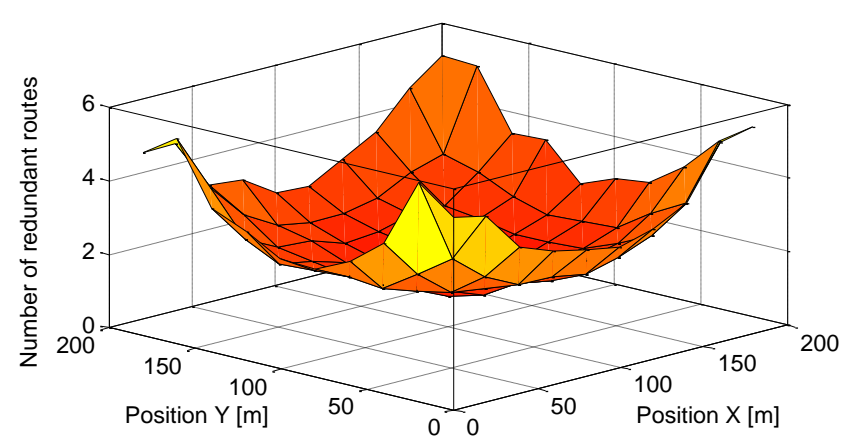

Fig. 10. Spatial distribution of the average number of redundant routes needed by MPAR (NonD approach) to establish a connection with $\mathrm{P}_{\text {req }}=0.999$ and $\delta_{\text {req }}=7$.

The use of multipath routes helps satisfying strict end-toend reliability and delay application requirements. However, redundant packet transmissions have a cost in terms of efficiency and capacity of the network. Fig. 11 represents the number of redundant packets received at the destination node for the same configuration used to obtain Fig. 9. Fig. 11 shows that MPAR results in that the same packet can be received in average at the destination node between 2 and 3 times. This is quite reasonable for the considered high application requirements.

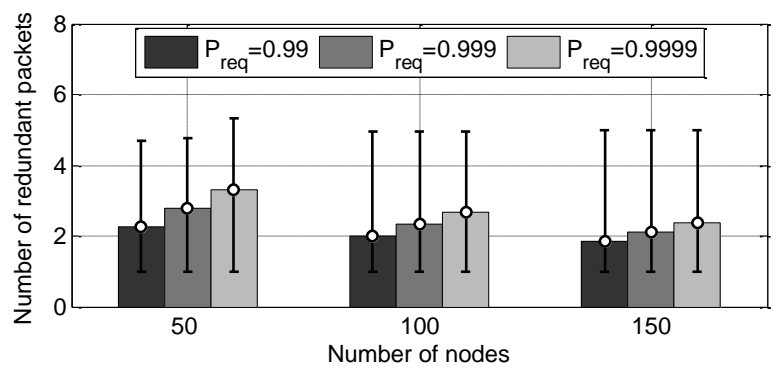

Fig. 11. Number of redundant packets received at the destination with MPAR (NonD approach) for $\delta_{\text {req }}=7$.

The previous figures were obtained considering only the end-to-end connections that were successfully established by the network manager using MPAR. The network manager does not establish an end-to-end connection when it detects that more than $R_{\max }$ routes are needed to satisfy the application requirements. Fig. 12 represents the percentage of connections established by MPAR as it detected that it was possible to meet the application QoS requirements. Fig. 12 shows that the percentage of established connections is especially low with a low number of nodes in the scenario and $R_{\max }$ equal to 7 . This is the case because of the higher distance between nodes that challenges the establishment of reliable wireless links. Fig. 12 also shows that relaxing the delay required by the application from 6 to 8 improves the possibility of establishing end-to-end connections. The results demonstrate that using MPAR, the network manager can detect beforehand whether it would be possible or not to establish an end-to-end connection that satisfies the application requirements. This MPAR feature helps avoiding trying to establish unreliable or unnecessary routes between source and destination.

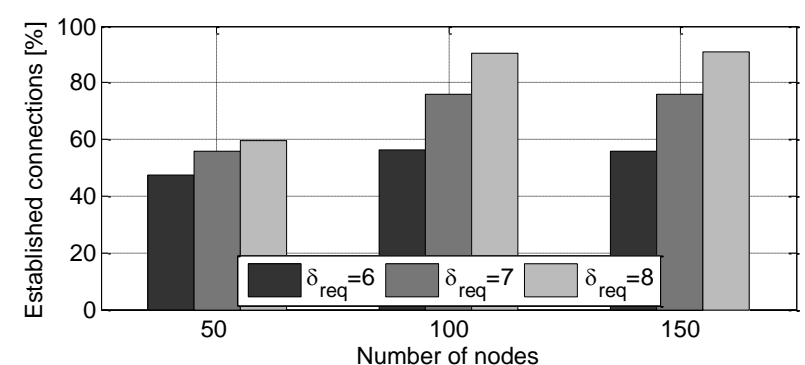

Fig. 12. Percentage of established connections by MPAR (NonD approach) that satisfy the QoS requirements considering $\mathrm{P}_{\text {req }}=0.999$.

Fig. 13 represents the spatial distribution of the percentage of established connections when $P_{\text {req }}=0.99$ and $\delta_{\text {req }}=7$. The figure shows that the nodes that cannot establish a connection with the network manager are normally those located close to the edges of the scenario. This is the case due their higher distance to the network manager (located at the center of the scenario), and therefore the lower probability to establish a reliable wireless end-to-end connection.

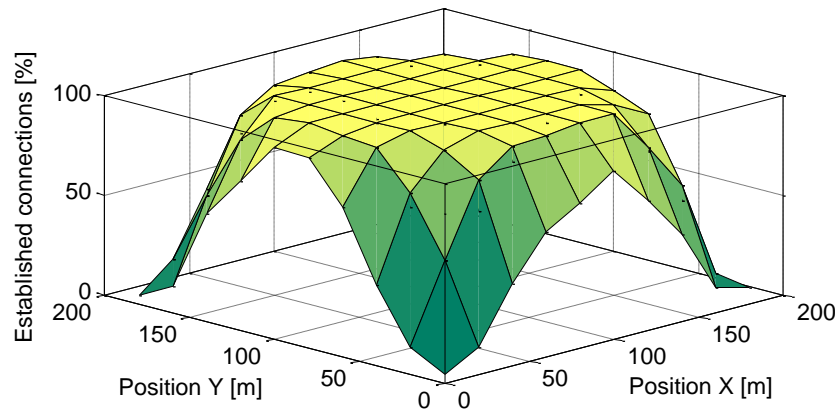

Fig. 13. Spatial distribution of the percentage of established connections with MPAR (NonD approach) that satisfy the QoS requirements $\left(\mathrm{P}_{\mathrm{req}}=0.999\right.$ and $\delta_{\text {req }}=7$ ).

All the previous results were achieved using the NonD approach defined within MPAR. The policy used by the network manager to create the multipath routes can have an impact on the load supported by each node and the possibility to satisfy the application QoS requirements. The NonD approach can create a multipath route that includes the same links and nodes in more than one route between source and destination. The LinkD and NodeD approaches avoid using the same links or nodes in different routes in an attempt to better distribute the load generated by a multipath route among the nodes of the network. However, LinkD and NodeD reduce the options (links and nodes) available to create the redundant routes, which can have an impact on the possibility to establish multipath routes capable to guarantee the QoS requirements of end-to-end connections. This effect is actually depicted in Fig. 14 that shows that NonD is the MPAR variant that results in the higher percentage of established connections that meet the application QoS requirements. 


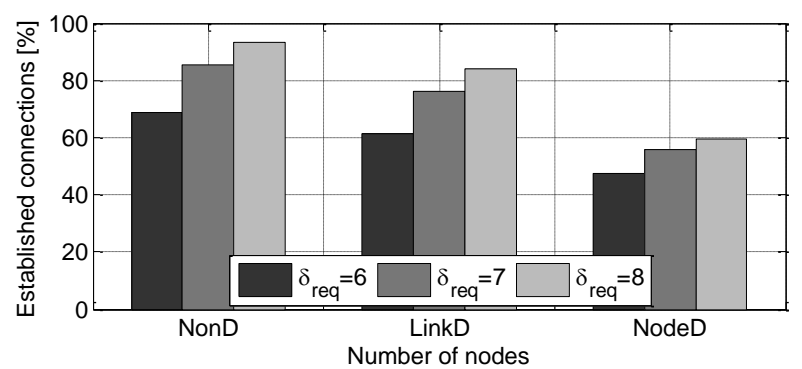

Fig. 14. Percentage of established connections that satisfy the QoS requirements considering 50 nodes and $\mathrm{P}_{\text {req }}=0.99$.

\section{Performance comparison}

This section compares the performance of MPAR to that obtained with different benchmark routing protocols. First, MPAR is compared against the QOLSR [11] single-path routing protocol that selects the optimum route between source and destination based on the bandwidth and delay. This comparison is aimed at highlighting the benefits of establishing multipath routes when strict application requirements have to be satisfied. For the comparison, we have adapted the QOLSR metrics to the scenario under study. The delay metric proposed in [11] is a function of the backoff time and the probability of packet collision. This relation is not valid in centralized TDMA networks like WirelessHART since they prevent packet collisions. The implemented scenario assumes that all links can provide the same bandwidth given that the network manager can assign to each connection a maximum of $n_{\max }$ slots per link in the simulated TDMA network. All these considerations required modifying the QOLSR scheme so that it can find the optimum route that minimizes the sum of the individual delays of each wireless link that makes up the multi-hop route from source to destination. The delay of each link is estimated with equation (8), and the optimum route is computed by the network manager using the Dijkstra algorithm. It is important noting that MPAR identifies and establishes the multipath routes that are capable to guarantee the application QoS requirements. On the other hand, QOLSR does not provide any guarantees but just establishes the identified optimum route between source and destination. This results in that QOLSR considerable reduces the percentage of established end-to-end connections that satisfy the reliability and delay requirements (Fig. 15). The direct comparison of Fig. 15 and Fig. 12 clearly demonstrates that MPAR is capable to establish a significantly higher percentage of end-to-end connections that can satisfy the application QoS requirements. Fig. 16 shows that the percentage of established connections by QOLSR that satisfy the QoS requirements rapidly decreases with the distance to the network manager. The gains achieved with MPAR are not only due to the establishment of multipath routes, but also to the fact that MPAR only establishes the routes that it estimates can guarantee with a high probability the application QoS requirements. On the other hand, QOLSR establishes an optimum route, but it cannot provide any QoS guarantees.

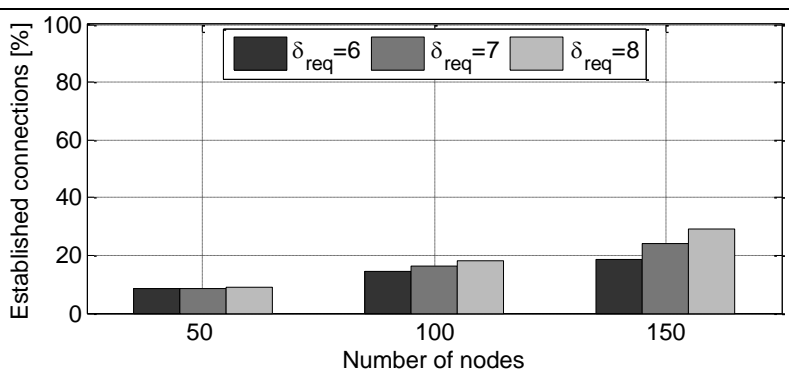

Fig. 15. Percentage of established connections by QOLSR that satisfy the QoS requirements considering $\mathrm{P}_{\text {req }}=0.999$.

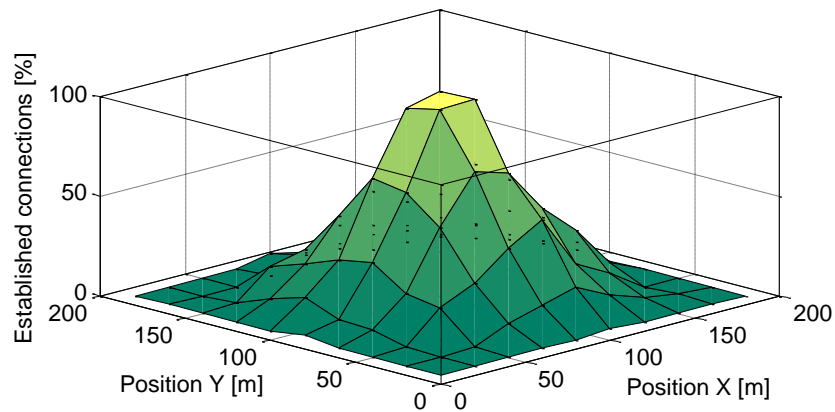

Fig. 16. Spatial distribution of the percentage of established connections by QOLSR that satisfies the QoS requirements $\left(\mathrm{P}_{\text {req }}=0.999\right.$ and $\left.\delta_{\text {req }}=7\right)$ considering 100 nodes in the network.

To demonstrate that the MPAR benefits are not only due to the establishment of multipath routes but also to its QoS guarantees, MPAR is also here compared with the multipath routing protocol proposed in [19]. This protocol pre-selects candidate paths during the routing discovery phase based on their bandwidth. The protocol uses RREQ and RREP messages to identify the routes in which the available bandwidth of all links is higher than the minimum required; the protocol excludes the routes with a number of hops higher than certain threshold. The available bandwidth is obtained at each link by measuring the channel busy time, i.e. the percentage of time that the channel is sensed as busy. Among the routes identified, those that provide higher reliability and are maximally disjoint (node disjoint) are preferred. [19] fixes the number of routes in a multipath route to 3 . This is done to achieve a compromise between load and network overhead in the discovery process. TDMA-based networks like WirelessHART can provide the same bandwidth to all routes if the same number of slots is assigned to each link of end-toend connections. The bandwidth of a route is then independent of the load of each link as long as all the necessary time slots can be allocated. Therefore, the application of the protocol proposed in [19] to the scenario under evaluation is equivalent to identifying the most reliable routes between source and destination. To this aim, the network manager exploits the PDR values collected from all links, and selects the 3 best routes in terms of reliability. For a fair comparison with MPAR using NonD, the proposal in [19] is also evaluated considering that routes can have nodes and links in common. Fig. 17 shows that the scheme proposed in [19] cannot always meet the reliability requirements for $\delta_{\text {req }}=7$. The end-to-end reliability obtained does not depend on the reliability demanded by the application, but is only influenced by the 
number of nodes in the scenario. Interestingly, high reliability levels can be obtained for 100 and 150 nodes because the probability of finding a reliable route is higher as the number of nodes increases. However, the most reliable routes are not necessarily the ones that minimize the delay or guarantee a delay below the threshold required by the application. In fact, Fig. 18 shows that the end-to-end delay requirements are not necessarily satisfied.

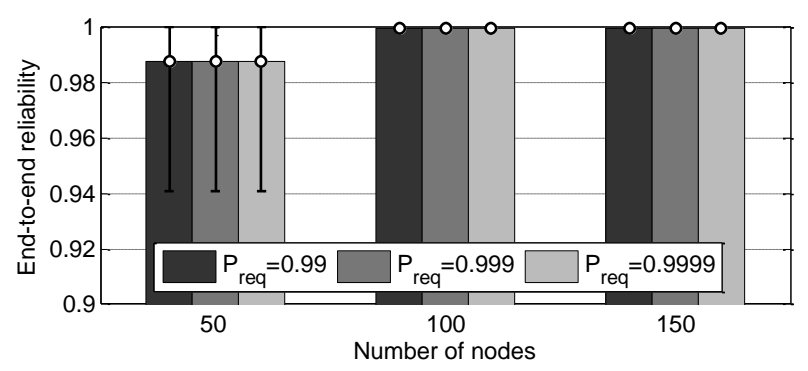

Fig. 17. End-to-end reliability experienced with the multipath routing protocol proposed in [19] for $\delta_{\mathrm{req}}=7$.

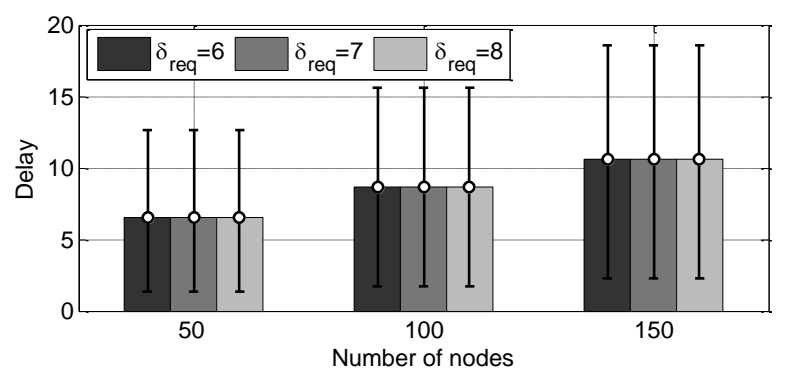

Fig. 18. End-to-end delay experienced with the multipath routing protocol proposed in [19] for $\mathrm{P}_{\mathrm{req}}=0.999$.

Finally, the performance of MPAR has also been compared with a routing protocol based on the WirelessHART standard. WirelessHART defines two routing methods, graph routing and source routing. However, the WirelessHART standard does not specify any routing algorithm, i.e. it does not define how the graphs are constructed for graph routing or how the routes are defined for source routing [6]. The routing protocol used here for comparison is based on graph routing and considers a primary route and an alternate route between the source and destination nodes. The primary route is used by default to transmit all packets, and supports multiple retransmissions on each hop $\left(n_{\max }\right)$. If the primary route fails, packets are transmitted through the alternate route, where no retransmissions are possible. This protocol is compatible with the WirelessHART standard and has been used in different studies as a reference [10][27][28]. For a fair comparison, the primary and alternate routes are built in this study using the same metrics than used in MPAR. In addition, they always consider dedicated links.

The reliability achieved with the implemented WirelessHART routing protocol does not depend on the reliability demanded by the application since the protocol creates the same number of redundant routes irrespectively of the application requirements. In addition, the reliability achieved with the implemented WirelessHART routing protocol strongly depends on the number of nodes. This is the case because the wireless link quality between nodes degrades with reducing number of nodes, and the number of redundant routes established by the WirelessHART routing protocol is fixed and does not take into account such degradation. In this case, a low number of nodes makes it difficult to find high quality routes, and the reliability achieved with the implemented WirelessHART routing protocol decreases.

Fig. 19 compares the end-to-end reliability achieved with the described WirelessHART routing protocol and MPAR for varying number of nodes ${ }^{3}$. The figure shows that MPAR achieves higher reliability levels than WirelessHART, even when MPAR is configured to meet the lowest reliability requirement simulated in this study $\left(P_{\text {req }}=0.99\right)^{4}$. Fig. 19 shows that the WirelessHART routing protocol cannot provide high reliability levels, especially for low number of nodes. On the other hand, MPAR can provide high reliability levels and satisfy the application's reliability requirements irrespectively of the number of nodes. It is interesting to note that the implemented WirelessHART routing protocol can achieve similar reliability levels to those provided by MPAR for 150 nodes and $P_{\text {req }}=0.99$. This is the case because when the number of nodes increases, the probability of finding reliable routes increases, and therefore the number of redundant routes required by MPAR is reduced. When the number of redundant routes established by MPAR approximates two, MPAR and the WirelessHART routing protocol achieve similar performance levels as they both utilize the same metrics for selecting routes and forwarding nodes. Additional simulations have been conducted for the WirelessHART routing protocol with $n_{\max }=2 . n_{\max }=2$ is equivalent to the default configuration in WirelessHART where only one retransmission per link is allowed in the primary route. Reducing the number of retransmissions in the primary route notably degrades the reliability achieved in the proposed scenario. In particular, the end-to-end reliability levels achieved with the implemented WirelessHART routing protocol and $n_{\max }=2$ are $0.66,0.85$ and 0.92 for 50,100 and 150 nodes, respectively.
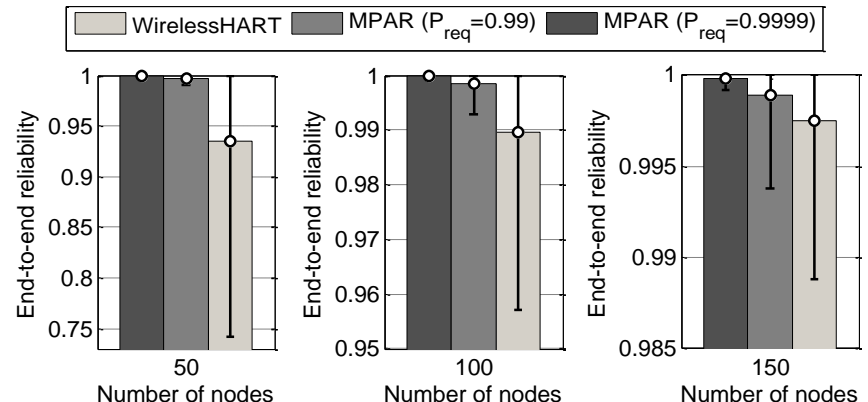

Fig. 19. End-to-end reliability experienced with the implemented WirelessHART routing protocol $\left(\mathrm{n}_{\max }=4\right)$ and with MPAR (NonD approach with $\delta_{\text {req }}=7$ ) for $\mathrm{P}_{\text {req }}=0.99$ and $\mathrm{P}_{\text {req }}=0.9999$.

\footnotetext{
${ }^{3}$ Please note the different scales in the y-axes of Fig. 19. In general, the reliability increases with the number of nodes; this effect was already pointed out in Fig. 5 and Fig. 7.

${ }^{4}$ As previously explained, MPAR adapts its configuration to achieve the reliability demanded by the application.
} 
Fig. 20 compares the delay experienced with the implemented WirelessHART routing protocol and MPAR. The figure shows that MPAR is able to produce lower end-toend delays than the implemented WirelessHART routing protocol. MPAR is also able to satisfy the delay requirements independently of the number of nodes and the requirements themselves. Fig. 20 depicts the delay performance of the implemented WirelessHART routing protocol when $n_{\max }=2$. If the number of allowed retransmissions is increased (e.g. $n_{\max }=4$ ), the implemented WirelessHART routing protocol improves its end-to-end reliability but also degrades its delay performance.

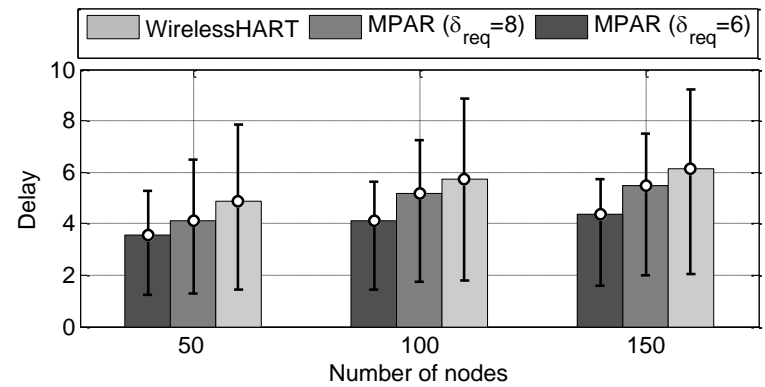

Fig. 20. End-to-end delay experienced with the implemented WirelessHART routing protocol $\left(\mathrm{n}_{\max }=2\right)$ and with MPAR (NonD approach with $\left.\mathrm{P}_{\mathrm{req}}=0.999\right)$ for $\delta_{\text {req }}=6$ and $\delta_{\text {req }}=8$.

\section{CONCLUSIONS}

Industrial wireless networks will play a critical role in the development of the Industry 4.0 concept and the factories of the future. This role necessarily requires industrial wireless networks capable to provide high reliability levels and low delays. To this aim, this paper has proposed MPAR, a multipath routing protocol that is able to guarantee the strict QoS levels demanded by industrial applications. MPAR utilizes probabilistic estimates of the reliability and delay of multipath routes to identify the nodes and routes needed to establish end-to-end connections that guarantee the demanded QoS levels. The MPAR proposal has been compared under realistic radio propagation conditions to other single-path and multipath routing protocols, and a routing protocol based on the WirelessHART standard. The obtained results demonstrate that the proposed multipath routing protocol improves the reliability and delay performance compared to existing single path and multipath routing protocols. The conducted study has also demonstrated that MPAR is capable to modify its configuration in order to satisfy the application requirements of wireless industrial applications under different scenarios. The efficiency of MPAR can also be observed in its capacity to detect situations in which the QoS levels cannot be guaranteed, and its capability to adapt the number of routes to the end-to-end connectivity conditions between source and destination and the application requirements. It is important noting that MPAR has been here evaluated considering industrial applications, and therefore strict end-to-end reliability and latency requirements. However, it can be adapted to consider other metrics and can be applied to other centralized TDMA-based multi-hop wireless networks.

\section{ACKNOWLEDGEMENTS}

This work was supported by the Spanish Ministry of Economy and Competitiveness and FEDER funds under the projects TEC2011-26109, TEC2014-5716-R and TEC201456469-REDT.

\section{REFERENCES}

[1] R. Drath, A. Horch, "Industrie 4.0: Hit or Hype?", IEEE Industrial Electronics Magazine, vol.8 (2), pp. 56-58, June 2014.

[2] A. Varghese, D. Tandur, "Wireless requirements and challenges in Industry 4.0", Proc. International Conference on Contemporary Computing and Informatics (IC3I), Mysore, India, pp. 634-638, 27-29 Nov. 2014.

[3] European Commission, "Factories of the Future PPP: towards competitive EU manufacturing", Research and Innovation - European Union 2013.

[4] A. Willig, "Recent and Emerging Topics in Wireless Industrial Communications: A Selection", IEEE Transactions on Industrial Informatics, vol. 4, pp. 102-124, May 2008.

[5] J.R. Gisbert et al., "Integrated system for control and monitoring industrial wireless networks for labor risk prevention", Journal of Network and Computer Applications, vol. 39, pp. 233-252, March 2014.

[6] IEC 62591 Ed. 1.0: Industrial communication networks -Wireless communication network and communication profiles-WirelessHART ${ }^{\mathrm{TM}}$, International Electrotechnical Commission, IEC, 2010.

[7] D. Chen, M. Nixon, and A. Mok, "WirelessHART Real-Time Mesh Network for Industrial Automation", Springer, 2010.

[8] J. Gozalvez, M. Sepulcre and J. A. Palazon, "On the Feasibility to Deploy Mobile Industrial Applications using Wireless Communications", Computers in Industry, vol. 65 (8), pp. 1136-1146, Oct. 2014

[9] J.M. Winter, G. Kunzel, I. Muller, C.E. Pereira, J.C. Netto, "Study of routing mechanisms in a WirelessHART network", Proc. IEEE International Conference on Industrial Technology (ICIT), Busan, Korea, pp. 1540-1545, 25-28 Feb. 2013.

[10] A. Saifullah, Y. Xu, C. Lu, Y. Chen, "End-to-End Communication Delay Analysis in Industrial Wireless Networks", IEEE Transactions on Computers, vol. 64 (5), pp. 1361-1374, May 2015.

[11] H. Badis and K. A. Agha, "QOLSR, QoS Routing for Ad Hoc Wireless Networks using OLSR", European Transactions on Telecommunications, vol. 15, no. 4, pp. 427-442, 2005.

[12] B. Carballido Villaverde, S. Rea, D. Pesch, "InRout - A QoS aware route selection algorithm for industrial wireless sensor networks", $A d$ Hoc Networks, vol. 10, pp. 458-478, 2012.

[13] J. Gebhardt, R. Gotzhein, A. Igel and C. Kramer, "QoS Multicast Routing in Partially Mobile Wireless TDMA Networks", Proc. IEEE Global Communications Conference (GLOBECOM), San Diego, CA, 610 Dec. 2015

[14] M. Wang and G.-S. Kuo, "An Application-Aware QoS Routing Scheme with Improved Stability for Multimedia Applications in Mobile Ad Hoc Networks", Proc. IEEE Vehicular Technology Conference (VTC-Fall), Dallas, USA, pp. 1901-1905, Sept. 2005.

[15] L. Cheng, J. Niu, J. Cao, S.K.. Das, G. Yu, "QoS Aware Geographic Opportunistic Routing in Wireless Sensor Networks", IEEE Transactions on Parallel and Distributed Systems, vol. 25 (7), pp. 18641875, July 2014.

[16] L. Oliveira, L. Almeida and P. Lima, "Multi-hop routing within TDMA slots for teams of cooperating robots", Proc. IEEE World Conference on Factory Communication Systems (WFCS), Palma de Mallorca (Spain), 27-29 May 2015.

[17] D. Ganesan, R. Govindan, S. Shenker and D. Estrin, "Highly resilient, energy-efficient multipath routing in wireless sensor networks", $A C M$ Mobile Computing and Communications Review, vol. 1 (2), Oct. 2002.

[18] A. Razzaque, M.M. Alam, M. Or-Rashid, C. S. Hong, "MultiConstrained QoS Geographic Routing for Heterogeneous Traffic in Sensor Networks", Proc. IEEE Consumer Communications and Networking Conference (CCNC), Las Vegas, USA, pp. 157-162, 10-12 Jan. 2008. 
[19] F. Qin and Y. Liu, "Multipath Based QoS Routing in MANET", Journal of Networks, vol. 4 (8), pp. 771-778, Oct. 2009.

[20] S.M. Mostafavi et al, "Delay minimisation in multipath routing using intelligent traffic distribution policies”, IET Communications, vol. 5 (10), pp. 1405-1412, 2011.

[21] A. Tsirigos and Z.J. Haas, "Analysis of Multipath Routing-Part I: The Effect on the Packet Delivery Ratio", IEEE Transactions on Wireless Communications, vol. 3 (1), pp. 138-146, January 2004.

[22] A. Tsirigos and Z.J. Haas, "Analysis of multipath routing-Part II: mitigation of the effects of frequently changing network topologies", IEEE Transactions on Wireless Communications, vol. 3 (2), pp. 500511, March 2004.

[23] A. Moussaoui, A. Boukeream, "A survey of routing protocols based on link-stability in mobile ad hoc networks", Journal of Network and Computer Applications, vol. 47, pp. 1-10, Jan. 2015.

[24] M. Plaschy, "Industry 4.0: wireless data acquisition systems and sensor networks, a key element for tomorrow's industry!", Feb. 2015. Online available: http://www.hikob.com

[25] EFFRA, "Overview of FP7-funded projects under the first call Developing Technologies for 'Factories of the Future"'. Online available: http://www.effra.eu

[26] E. Tanghe, et al., "The industrial indoor channel: large-scale and temporal fading at 900, 2400, and $5200 \mathrm{MHz}$ ", IEEE Transactions on Wireless Communications, vol. 7 (7), pp. 2740-2751, July 2008.

[27] A. Saifullah et al., "Schedulability Analysis under Graph Routing in WirelessHART Networks", Proc. IEEE Real-Time Systems Symposium (RTSS), San Antonio, pp. 165-174, 1-4 Dec. 2015.

[28] M. Nobre I. Silva and L. A. Guedes, "Routing and Scheduling Algorithms for WirelessHARTNetworks: A Survey", Sensors, vol. 15(5), pp. 9703-9740, 2015.

Miguel Sepulcre (msepulcre@umh.es) received a Telecommunications Engineering degree in 2004 and a Ph.D. in Communications Technologies in 2010, both from the University Miguel Hernández of Elche (UMH), Spain. He was awarded by the COIT (Spanish official association of Telecommunication Engineers) with the ONO prize to the best national Ph.D. He has been visiting researcher at the European Space Agency (ESA) in Noordwijk (The Netherlands) in 2004, at the Karlsruhe Institute of Technology (KIT) in Karlsruhe (Germany) in 2009, and at Toyota InfoTechnology Center in Tokyo (Japan) in 2014. He serves as Associate Editor for the IEEE Vehicular Technology Magazine, and is part of the Editorial Board of the IEEE Connected Vehicle Initiative. He was Publicity Co-Chair in ACM VANET in 2012 and 2013, track-chair in IEEE VTC-Spring 2015 and IEEE VTC-Fall 2011 and was part of the organizing committee of URSI 2012 (Spanish national symposium of the international union of radio science). He is now Assistant Professor at the Communications Engineering Department of UMH, and member of UWICORE research laboratory working in wireless vehicular and industrial networks.

Javier Gozalvez (j.gozalvez@umh.es) received an electronics engineering degree from the Engineering School ENSEIRB (Bordeaux, France), and a $\mathrm{PhD}$ in mobile communications from the University of Strathclyde, Glasgow, U.K. Since October 2002, he is with the Universidad Miguel Hernández de Elche, Spain, where he is currently an Associate Professor and Director of the UWICORE laboratory. At UWICORE, he leads research activities in the areas of wireless industrial networks, multi-hop cellular networks, vehicular networks, resource management and heterogeneous networks. He has published over 125 papers in international conferences and journals. He has received several awards at international and national conferences, the best research paper award from the Journal of Network and Computer Applications (Elsevier) in 2014, and the Runner-up prize for the "Juan López de Peñalver" award of the Royal Academy of Engineering in Spain that recognizes the most notable Spanish engineers aged below 40 . He is an expert evaluator for the European Commission and research agencies across Europe. He is an elected member to the Board of Governors (2011-2016) and 2016 President of the IEEE Vehicular Technology Society (IEEE VTS). He served as IEEE Distinguished Lecturer for the IEEE VTS, and currently serves as Distinguished Speaker. He currently serves as the Chair of the IEEE Connected Vehicles initiative. He serves as Mobile Radio Senior Editor of the IEEE Vehicular Technology Magazine, and on the Editorial Board of the Computer Networks journal. He was the General Co-Chair for the IEEE VTCSpring 2015 conference in Glasgow (UK), ACM VANET 2013, ACM VANET 2012 and 3rd ISWCS 2006. He also was TPC Co-Chair for 2011 IEEE VTC-Fall and 2009 IEEE VTC-Spring. He was the founder and General
Co-Chair of the IEEE International Symposium on Wireless Vehicular communications (WiVeC) in its 2007, 2008, and 2010 editions.

Baldomero Coll-Perales (bcoll@umh.es) received a Telecommunications Engineering degree in 2008 and a Ph.D. in Industrial and Telecommunications Technologies in 2015, both from the Miguel Hernandez University (UMH) of Elche, Spain. He received Best Student awards in Telecommunications Engineering both by UMH and the professional organization of Telecommunications Engineers. In April 2010, he obtained a Ph.D. fellowship from the Valencia regional government and joined the UWICORE research laboratory to work on the development of networking and communication protocols for multi-hop cellular systems using mobile relays under the Opportunities, m-HOP and ICARUS projects. As part of his thesis, in 2012 he spent three months at the Institute of Telecommunications of King's College London (UK) working on the design of efficient opportunistic multi-hop cellular networks. He is currently a research fellow at UWICORE working on multi-hop cellular networks, including networking, connectivity and resource management aspects under the 5GEAR project. 